\title{
La historia en su encerramiento: Una mirada iconoclasta al quehacer de la historia en Colombia
}

\author{
Alberto Guillermo Flórez Malagón \\ Pontificia Universidad Javeriana \\ Instituto de Estudios Sociales y Culturales, Pensar
}

\begin{abstract}
Resumen
Este artículo sitúa la discusión sobre la historia en el contexto de la crisis disciplinal experimentada actualmente por las ciencias sociales y humanas. Se evidencia que la historia no posee un estatuto diferente frente a la crisis y por lo tanto su desarrollo debe estudiarse a la luz de la revolución epistemológica más general. Se introduce la idea de la transdisciplinariedad como una de las alternativas de reconstrucción del conocimiento y como uno de los campos más promisorios: el de los estudios culturales, considerando incluso que la historia podría concebirse como una versión de este nuevo campo.
\end{abstract}

\begin{abstract}
This article discusses history as a discipline in the context of the epistemological crisis currently experimented by the social sciences and the humanities. This discussion is possible because history does not have a special status facing the crisis as we present it. Introducíng the idea of transdisciplinarity and its expression in the form of cultural studies leads us to propose a new understanding of history as a version of this new field.
\end{abstract}

Alberto Guillermo Flórez Malagón. "La Historia en su Encerramiento. Una mirada iconoclasta al quehacer de la historia en Colombia". (La versión original de este artículo fue presentada como ponencia en el panel La Crisis de la Historia (social) y los Retos del Historiador al Comienzo de un Nuevo Milenio, en el XI Congreso Colombiano de Historia, Universidad Nacional de Colombia, Bogotá, agosto de 2000). 


\section{FRONTERAS}

\section{Introducción}

El desarrollo actual de las ciencias sociales y humanas se sitúa en un terreno abonado para nuevas formas de pensamiento. La revolución "científica" que experimentamos actualmente da salidas a nuevas preguntas, a nuevas formas de conocer, formas complejas, holísticas y transdisciplinarias. Inevitablemente, a pesar de las fuertes resistencias, se están desarrollando nuevas ciencias sociales, nuevos campos de pensamiento y conocimiento, y por lo tanto nuevas formas de acción.

Uno de los principales puntos de ruptura, que potencia esta revolución del pensamiento, ha sido aquel de la disciplinariedad y su jerarquía interna, es decir el de la fragmentación de los saberes y la jerarquización de los mismos a partir de modelos científicos que, además, despojaron al conocimiento de sus elementos constitutivos, éticos y estéticos.

Las críticas internas y externas al quehacer disciplinar tratan recurrentemente del "fin" de las disciplinas, o por lo menos del fin de sus cánones como estaban planteados hasta ahora. Esto al punto de pensarse, como lo ha expresado de manera tajante el conocido sociólogo inglés Anthony Giddens, que las líneas de pensamiento que dividen a los académicos contemporáneos son solamente mitos. ${ }^{1}$

Así, el debate actual, el cual seguirá definiendo el futuro cercano de las humanidades y las ciencias sociales, expresa la defensa cerrada de la disciplinariedad clásica en un extremo y la negación de su existencia, en el otro. En el proceso concreto se reconocen muchas opciones intermedias que responden a esta polarización. Aparecen nuevas subdisciplinas y nuevos arreglos disciplinares e interdisciplinares, pero también nuevos campos antidisciplinares, y se plantean disciplinas híbridas y opciones transdisciplinares. Todo ello expresa niveles de reconfiguración que dependen del grado de radicalidad al cual se lleve la crítica ante la crisis de fundamentación.

En este texto referiré el debate más general entre disciplinariedad y transdisciplinariedad que ha sido tan influyente en los últimos años para plantear algunas intuiciones acerca de su impacto sobre el quehacer histórico.

${ }^{1}$ Anthony Giddens, "Classical Social Theory and the Origins of Modern Sociology," American Journal of Sociology 81 (Enero 1996): 703-29. 
Esto a pesar de que los historiadores en general no han sido fuertemente permeados por dicho debate, ya sea por la recia profesionalización que han sufrido en todas las academias del mundo, ya sea porque sus practicantes sienten que heredaron una tradición de miles de años, y quizás más importante porque su estatus siempre se ha camuflado entre las ciencias sociales y las humanidades, eludiendo a menudo las preguntas críticas acerca del estatuto disciplinar.

En el caso colombiano lo que aparece como dominante en la mayor parte de las discusiones acerca del estatus de la historia se limita casi exclusivamente al tema del desarrollo disciplinar: se estudian las escuelas, las tendencias, y hasta se han ido acomodando las transformaciones más radicales originadas en el famoso giro lingüístico y en las propuestas de la historia posmodernista, pero muy pocas veces se involucra a la historia en la discusión más amplia acerca de la crisis y reconstitución disciplinares en contextos no disciplinarios. ${ }^{2}$

Aquí sugeriré una reflexión, con visos regionales, que permita ir abriendo el espacio de los historiadores hacia estas nuevas preguntas que se resuelven ya no en el campo de la interdisciplina (relacional) o de la multidisciplina (asociativa) sino en el de la transdisciplína (integradora y holística), propuesta esta última que afecta el estatus de todas las disciplinas incluyendo la historia, hasta ahora confortablemente protegida entre los muros de su aparentemente sólido oficio.

\footnotetext{
${ }^{2}$ Interesantes aproximaciones críticas al quehacer "interno" de la disciplina aparecen por ejemplo en Carlos Miguel Ortiz Sarmiento y Bernardo Tovar Zambrano, eds., Pensar el Pasado (Santafé de Bogotá: Archivo General de la Nación, Universidad Nacional de Colombia, 1997), o en Mauricio Archila, "¿Es Aún Posible la Búsqueda de la Verdad? Notas sobre la (Nueva) Historia Cultural," Anuario Colombiano de Historia Social y de la Cultura (en adelante citado como ACHSC) 26 (1999): 249-83, un artículo bastante "avanzado" en la discusión si se compara con las recientes y aún tradicionales revisiones historiográficas como la provocadora y muy "moderna" escrita por Jesús Antonio Bejarano (q.e.p.d.), "Guía para Perplejos: Una Mirada a la Historiografía Colombiana," ACHSC 24 (1997): 283-329 y la muy juiciosa de Jorge Orlando Meló publicada en la Revista de Estudios Sociales 4 (1999) de la Universidad de los Andes. Sin embargo, la pregunta acerca de una posible disolución de las fronteras disciplinares apenas se insinúa allí pues los autores miran la crisis de la disciplina desde adentro sin permitirse explorar alternativas más radicales como las que aquí se proponen y las cuales implican tomar con menos prevenciones el ambiente crítico del posmodernismo.
} 


\section{El agotamiento de las disciplinas. ¿ Reconfiguración o desaparición?}

Entre 1993 y 1996 un grupo de influyentes pensadores provenientes de diversas formaciones disciplinares y regiones del mundo se reunió para conformar la Comisión Gulbenkian. ${ }^{3}$ Este notable grupo realizó un análisis que, traducido a varias lenguas, se ha convertido en una referencia clásica para el estudio del desarrollo de las ciencias sociales y las humanidades, y de su estado actual, tanto en términos de las relaciones entre sus disciplinas como en sus relaciones con las ciencias naturales.

El reporte de esta comisión exploró cómo las ciencias modernas se construyeron históricamente y por qué fueron fragmentadas en un grupo de disciplinas que hoy en día están siendo retadas como referencias adecuadas para la división del trabajo intelectual creando, en consecuencia, un importante debate alrededor de su estructura organizacional. Esta preocupación coincide, parcialmente, con la crítica más amplia que han planteado las corrientes posmodernistas ante el desarrollo y la legitimidad del conocimiento científico. Aunque la "aventura del posmodernismo", como la describe Carlos Reynoso ${ }^{4}$, es más conocida por la línea que tiene que ver con la literatura, la filosofía y el arte que con aquella que discute las ciencias sociales, en ambos casos su aproximación más generalmente aceptada ha decretado (entre otras cosas) la crisis de la razón y de la ciencia partiendo de un fuerte escepticismo hacia sus categorías analíticas.

Las ciencias sociales se encontraron finalmente definidas entre 1850 y 1914 en estructuras disciplinares reconocidas por las universidades del mundo, presas en la mitad de la tensión entre las ciencias de la naturaleza y las ciencias del espíritu, como las refería la discusión neokantiana, división que más tarde asumirían las corrientes positivistas. ${ }^{5}$ Pero, como diría Jürgen Habermas, "basta dar una ojeada a cualquier catálogo de cursos universitarios para percatarse de esta escisión fáctica

\footnotetext{
${ }^{3}$ Financiada por la Fundación Calouste Gulbenkian, esta comisión reunió a seis especialistas de las ciencias sociales, dos de las ciencias naturales y dos de las humanidades. Ellos fueron: Immanuel Wallerstein, Calestous Juma, Evelyn Fox Keller, Jürgen Kocka, Dominique Lecourt, V. Y. Mudimbe, Kinhide Mushakoji, Ilya Prigogine, Peter J. Taylor y Michel-Rolph Trouillot.

${ }^{4}$ Carlos Reynoso, comp., El Surgimiento de la Antropología Posmoderna (Barcelona: Gedisa, 1992), 11.

${ }^{5}$ Jürgen Habermas, Las Ciencias Sociales (Barcelona: Tecnos, 1987), 81.
} 
de las ciencias que sólo los manuales de los positivistas parecen considerar irrelevante". ${ }^{6}$ Las ciencias sociales jamás han podido ser indiferentes a esta tensión, mientras que las otras ciencias, definidas en los extremos de las "dos culturas" parecen ignorarse mutuamente desde una perpectiva epistemológica (la teoría de la ciencia y la hermeneutica filosófica).

Como nos lo recordó Michel Foucault, uno de los principales orientadores de las corrientes posestructuralistas (vanguardia del pensamiento posmoderno), no podemos ignorar más la arbitrariedad de las epistemes y sus implicaciones sobre el tema de la fundamentación disciplinar. Además, "las disciplinas constituyen un sistema de control en la producción del discurso, fijando sus límites a través de la acción de una identidad que toma la forma de una permanente reactivación de las reglas".' En esta línea de pensamiento resulta indispensable la pregunta acerca de la fundamentación disciplinar y de su proyección hacia una política del saber.

Por otra parte, los grandes logros intelectuales de los últimos cuarenta años, los cuales nos han guiado hacia las ciencias de la complejidad (que algunos prefieren referir como teorías del caos), así como la necesidad que surgió para la "contextualización" de los universalismos, el entendimiento de la poscolonialidad, de la subalternidad y sobre todo de la globalización ${ }^{8}$, han influenciado la práctica de los académicos humanistas pero, desafortunadamente, han dejado poco espacio para preocupaciones de naturaleza estructural y organizativa. En el presente estado de su evolución parece que este último tema, cuyo referente principal es la estructura disciplinar y su institucionalización, debe considerarse un dilema central para las ciencias sociales y las humanidades. ${ }^{9}$

Recordando de manera referencial el lenguaje de Thomas Kuhn, podríamos estar asistiendo a una revolución científica que surge de una doble debilidad de las

\footnotetext{
${ }^{6}$ Ibid.

${ }^{7}$ Michel Foucault, The Archaeology of Knowledge and the Discourse on Language (New York: Phanteon, 1972), 224, citado en Immanuel Wallerstein et al., Abrir las Ciencias Sociales (México: Siglo XXI, 1998), 33.

${ }^{s}$ Acerca de la complejidad de estas nuevas problemáticas, en referencia a América Latina véase Santiago Castro G. y Eduardo Mendieta, coord., Teorías sin Disciplina: Latinoamericanismo, Poscolonialidad y Globalización en Debate (México: Universidad de San Francisco, Grupo Editorial Porrúa, 1998).

${ }^{9}$ Wallerstein et al., Abrir las Ciencias Sociales, x.
} 
ciencias sociales: por defecto (ningún paradigma dominante) y por exceso (varias propuestas paradigmáticas en competencia). La crisis no sólo plantea el desdibujamiento de los objetos disciplinares en las ciencias sociales sino también la conexión de los modelos de las ciencias naturales con los sociales, o por lo menos con los sistemas no lineales, análogos a los sociales.

Aunque la realidad no existe fragmentada, los pensadores disciplinares así la representaron. Las disciplinas siguieron sus propios lenguajes, cultura de investigación y aplicaciones prácticas. La dificultad radica en que, a medida que ellas se especializaron, cesaron de ver, o aún prever las conexiones potenciales que ellas tienen con otras áreas. ${ }^{10}$

La primera salida práctica, casi pragmática, que se planteó ante estos problemas, nos remitió a la idea de la interdisciplinariedad y a la constatación de su tremendo auge metodológico ${ }^{11}$ Muy intensamente a partir de 1945 las restricciones disciplinares se empezaron a romper. A lo largo del mundo las series de coloquios y conferencias internacionales en décadas recientes han tendido a reclutar participantes de acuerdo con temas específicos, la mayor parte de las veces sin poner demasiada atención a las afiliaciones disciplinares. Hoy en día existe un número creciente de importantes revistas científicas que conscientemente ignoran las fronteras disciplinares. Los departamentos disciplinares recluían a sus profesores en una variedad amplia de profesiones. Y por supuesto existe un auge de nuevos programas cuasidisciplinares que se han consolidado en la última mitad del siglo y que están generalmente conformados por personas que poseen grados universitarios en múltiples disciplinas. Estas prácticas académicas se han consolidado con mucha naturalidad y aparecen como dominantes en la actividad investigativa contemporánea.

Aunque antes de 1937 se habló de cruce de disciplinas y hasta de demolición de las fronteras disciplinares, el término interdisciplinariedad aparece en ese año mencionado por primera vez por el sociólogo Louis Wirtz. ${ }^{12}$ El desarrollo de esta experiencia,

\footnotetext{
${ }^{10}$ Ibid.

${ }^{11}$ Ver Mattei Dogan, "Disgregación de las Ciencias Sociales y Recomposición de las Especialidades" (Marzo 1994, mimeografía).

${ }^{12}$ Ver David L. Sills, "A Note on the Origin of Interdisciplinarity," ITEM (Social Science Research Council, marzo 1986).
} 
especialmente después de la Segunda Guerra Mundial, llevó sin embargo al sentimiento de una simple e insuficiente amalgama en la que aparecía un desfile de disciplinas, en el mejor de los casos creando apenas un paralelismo útil. Un famoso documento de la Organización de Cooperación y Desarrollo Económico llegó incluso a tildar esas experiencias como un fracaso, refiriendo a la interdisciplinariedad como un concepto epistemológicamente ingenuo. ${ }^{13}$ Los enfoques interdisciplinarios y multidisciplinarios así vistos resultaban engañosos, además porque insistían en la división de la realidad en diversos fragmentos.

La pluridisciplinariedad como otra expresión análoga consistiría en el estudio del objeto de una sola y misma disciplina por medio de varias disciplinas a la vez. Dicho de otro modo, la gestión pluridisciplinaria sobrepasaría las disciplinas pero su finalidad quedaría igualmente inscrita en el marco de la investigación disciplinaria. La interdisciplinariedad, a su vez, tenía una mirada diferente concerniente a la transferencia de métodos de una disciplina a otra. Todas estas prácticas sobrepasaban a las disciplinas pero su finalidad quedaba de todas maneras inscrita en la investigación disciplinaria.

Esta "zona de libre comercio" , como llama Nelly Richard a la suma pacífica de saberes complementarios (interdisciplinariedad, multidisciplinariedad $\mathrm{o}$ pluridisciplinariedad), no sería más que un ordenamiento de saberes destinados a integrar una nueva totalidad de conocimientos más abarcadora y funcional que finalmente dejaría intocados los contornos de cada saber heredado. ${ }^{14}$

En la evolución más reciente del problema no se tiende a hablar tanto de síntesis como de hibridación, lo cual denota en algunos casos recombinación de fragmentos de ciencias, planteando el cruce y reconstitución de las disciplinas de dos en dos. Pero también se habla, quizás de manera aún más útil, del término transdisciplinariedad, en el cual, se reconoce el origen múltiple de nuevos campos de conocimiento y la imposibilidad del trabajo monodisciplinario y aun interdisciplinario para absorber las nuevas temáticas desarrolladas por el mundo contemporáneo.

\footnotetext{
${ }^{13}$ OCDE, Interdisciplinary Problems of Teaching and Research in Universities (París: OCDE, 1972).

${ }^{14}$ Nelly Richard, "Antidisciplina, Transdisciplina y Redisciplinamientos del Saber," Revista de Estudios Sociales 1 (agosto 1998): 118-23, de la Universidad de los Andes y la Fundación Social.
} 


\section{FRONTERAS}

Potenciada por la crítica posmodernista, esta veta de ruptura se ha convertido en una alternativa que aboga por un conocimiento más allá de las disciplinas. Por una mirada integradora, comúnmente tratada como holística. La transdisciplinariedad no busca el dominio en varias disciplinas sino abrir todas las disciplinas a lo que todas tienen en común y a lo que yace más allá de sus fronteras. La visión transdisciplinaria es además definitivamente abierta en cuanto trasciende el campo de las ciencias exactas estimulándolas para que se comuniquen y reconcilien, no únicamente con las humanidades y las ciencias sociales sino también con el arte, la literatura, la poesía y otras experiencias espirituales. ${ }^{15}$

¿Hay algo entre y a través de las disciplinas y más allá de toda disciplina? Desde el punto de vista del pensamiento clásico no hay nada, estrictamente nada. El espacio en cuestión está vacío, como el vacío de la física clásica. Aun cuando renuncia a la visión piramidal del conocimiento, el pensamiento clásico considera que cada fragmento de la pirámide disciplinaria es una pirámide entera; cada disciplina pretende que el campo que le pertenece es inagotable. Para el pensamiento clásico la transdisciplinariedad es un absurdo porque no tiene objeto. En cambio para la transdisciplinariedad el pensamiento clásico no es absurdo pero su campo de aplicación es considerado restringido.

La investigación disciplinaria concierne más o menos a un único y mismo nivel de realidad; por otra parte, en la mayoría de los casos no concierne más que a los fragmentos de un solo y mismo nivel de realidad. En cambio la transdisciplinariedad se interesa en la dinámica que se engendra por la acción simultánea de varios niveles de realidad. ${ }^{16}$

Así, como dicen algunos, las ciencias sociales y las humanidades están genéticamente programadas para dar a luz a mestizos ${ }^{17}$ y estos no son simples Frankensteins de las ciencias sociales y las humanidades, sino cuerpos completos de conocimiento en los cuales, además, se están resolviendo los problemas más relevantes a las

\footnotetext{
${ }^{15}$ Lima de Freitas, Edgar Morin y Basarab Nicolescu, "Declaración Transdisciplinaria" (documento elaborado por el Comité de Redacción, Primer Congreso Mundial sobre Transdisciplinariedad, Convento da Arrábida, Portugal, 6 de noviembre de 1994).

${ }^{16}$ Ibid., artículos 3 y 5.

${ }^{17}$ Dogan, "Disgregación de las Ciencias."
} 
culturas y a las sociedades contemporáneas. ${ }^{18}$ Así, la transdisciplina no busca un espacio de convergencias e hibridación, sino una etapa superior, por lo menos un estadio diferente del de las disciplinas y del de la interdisciplina.

Frente a la transdisciplina surge un debate importante el cual remite al enfrentamiento entre la propuesta totalizante de la modernidad y las visiones posmodernas más radicales, antidisciplinares si se quiere, que reivindican una diversidad y fragmentación de las situaciones objeto de conocimiento, lo cual impediría una propuesta reconstructiva y por supuesto negaría la vuelta a cualquier tipo de metarrelato. ${ }^{19}$ Personalmente, considero que el metarrelato podría estar presente en las prácticas transdisciplinares sin que ello afecte su carácter revolucionario en cuanto a la epistemología de las ciencias se refiere. Sin embargo, las opciones que ofrecen los defensores de la transdisciplinaridad expresan una tensión permanente acerca de estos temas, presente en los nuevos campos. ${ }^{20} \mathrm{~A}$ este nivel aparece el problema de si los nuevos campos simplemente redefinen fronteras que seguirán inevitablemente siendo artificiales e inadecuadas.

En todo caso, las alternativas expresadas en los nuevos campos transdisciplinares del conocimiento son inmensas. Todos comparten la angustia de querer tratar de manera diferente los problemas que no parecen resolverse adecuadamente desde la ciencia disciplina!. Todos son explícitamente políticos con un claro horizonte moral.

\footnotetext{
${ }^{18}$ En este punto recuerdo la coincidencia con la reflexión historiográfica de Medina cuando insiste en la responsabilidad del historiador frente a "un país concreto" (el aquí y el ahora), lo cual parece expresar la necesidad de vincular el conocimiento a la cotidianidad en un intento ético-político de reconstrucción disciplinar. Medófilo Medina, "La Historia: Hic et Nunc," en Pensar el Pasado, 51-1 A. ${ }^{19}$ Roberto Folian, Problematizing Interdisciplinarity," en Social Sciences and Transdisciplínarity: Latin American and Canadian Experiences, ed. Rosalind Boyd y Alberto Flórez Malagón (Montreal: CDAS, 1999), 11-116

${ }^{20}$ Existen puntos tan opuestos como los expresados por Bunge y Alfonso Borrero, S.J., quienes hablan de interdisciplinariedad y ocasionalmente de transdisciplinariedad desde marcos cientificistas clásicos, equivaliendo estas propuestas a simples cruces entre conjuntos (intersecciones en lenguaje matemático), mientras otros como Roberto Follari previenen acerca de la "tentación totalizante" que implica el auge de los nuevos campos. Véase Mario Bunge, "Sistemism: The Alternative to Individualism and Holism," en Social Sciences and Transdisciplinarity; Alfonso Borrero, S.J., "La Interdisciplinariedad: Concepto y Práctica" (ponencia presentada al Simposio Permanente sobre la Universidad, XX Seminario General Nacional, Universidad Javeriana, Bogotá, 2000) y Follari, "Problematizing Interdisciplinarity," en Social Sciences and Transdisciplinarity.
} 


\section{FRONTERAS}

Y, además, han tenido alto éxito en sus procesos de institucionalización. Los estudios ambientales, de cultura visual, de género, culturales, poscoloniales y subalternos, son algunos de los ejemplos más conocidos.

Los nuevos campos aparecen cuando se deshace la solidaridad de las antiguas disciplinas en provecho de un objeto nuevo, de un lenguaje nuevo. Es aquí donde surge la posibilidad de insistir en la vocación política ${ }^{21}$ de los nuevos campos para insistir en un nuevo proyecto "democratizador de transformación académica" que permita leer ya no "nuevos objetos" u "objetos justos" sino la posibilidad de nuevos lenguajes, nuevos registros de conocimiento y también de escritura.

Esta reconfiguración política expresa también importantes transformaciones en el desarrollo institucional de las universidades y demás centros educativos, cambios que se relacionan directamente con los contenidos sociales, económicos y culturales de la acción guiada y legitimada por el conocimiento.

No es gratuito que el problema de las fronteras disciplinares sea cada vez más una excusa para la reorganización institucional de los saberes. Desde las primeras tendencias interdisciplinarias, hasta las más recientes transdisciplinarias, con el auge de los nuevos campos, las universidades como los principales centros académicos del mundo, empiezan a adaptarse a las nuevas tendencias, aunque sin lograr todavía definir del todo su nueva ideología.

La dificultad por transformar la Universidad actual radica en que las nuevas propuestas, como la transdisciplinariedad, van más allá de una simple reconstitución de saberes. El tema se ha convertido en uno de sus compromisos. Cuando las viejas disciplinas (especialmente la historia, la antropología y la literatura) se preguntan por qué los nuevos campos las superan, o por lo menos están más allá de sus propuestas, parte de la respuesta radica en su actitud política.

Aunque todas las expresiones de las nuevas ciencias son complejas y podrían ser discutidas en cada caso particular, aquí privilegiaremos una de estas opciones, los

\footnotetext{
${ }^{21}$ John Beverly, "Write in Reverse on the Projects of the Latín American Subaltern Group," Disposition (Universidad de Michigan, 1994), xxi, citado en Richard, "Antidisciplina, Transdisciplina," 122.
} 
estudios culturales, porque representan una fuerza renovadora muy importante en toda la América Latina, porque en el caso colombiano su evolución ha sido muy dinámica y ya comienza incluso a institucionalizarse en varias de las principales universidades colombianas y sobre todo porque su carácter crítico resulta muy pertinente para aportar académicamente a la discusión sobre la crisis de la historia.

Los estudios culturales optan por estudiar la cultura contemporánea como un objeto más amplio que cualquiera que hubieran propuesto la antropología, la historia o la crítica literaria. Este sería un buen ejemplo de reconfiguración de las disciplinas alrededor de una propuesta de hibridación, en la cual se percibe no tanto la reacomodación de la disciplinariedad moderna, sino la recuperación de un objeto complejo que pudo perderse en el afán hegemonizante de la ciencia.

\section{Como sintetizan Santiago Castro y Oscar Guardiola,}

"la novedad de este campo emergente radica en el abordaje postradicional que hacen de su objeto de estudio. La cultura ya no es vista como un conjunto "orgánico" de valores, lenguajes, mitos y creencias que se transmiten de generación en generación y que definen la identidad de un pueblo (concepto "antropológico" de cultura). La "cultura" que estudian los estudios culturales no es letrada, ni depende del conocimiento (Bildung) de los sujetos (concepto "humanista" de cultura). Tampoco se trata de un mero epifenómeno de las determinaciones económicas de la base (concepto "economicista" de cultura). En el origen del proyecto de los estudios culturales se encuentra pues una crítica triple: del organicismo, del humanismo y del economicismo de las ciencias sociales.

En una palabra: la "cultura" de la que se ocupan los estudios culturales tiene menos que ver con los artefactos culturales en sí mismos (textos, obras de arte, valores, tecnologías, conocimientos, etc.), como con los procesos estructurales de producción, distribución y recepción de esos artefactos... Los estudios culturales toman como objeto de análisis esos mecanismos de producción de significados, el modo en que se reproducen y distribuyen, los dispositivos de selección que utilizan, así como la forma en que se genera la dialéctica entre sujeto y estructura" ${ }^{22}$ 
La principal tarea de los estudios culturales es, entonces, la de enfocarse en el estudio de la función política de la cultura. En otras palabras, estudiar las prácticas culturales y sus relaciones con el poder. De allí que su preocupación constante sea exponer las relaciones de poder y examinar cómo estas relaciones influencian y moldean las prácticas culturales. Este enfoque crea una diferencia con los estudios de la cultura que desarrollaron las disciplinas clásicas, en los cuales se concebía la cultura como una entidad discreta divorciada de su contexto social o político. Además, es muy importante que los estudios culturales insisten en no separar la experiencia subjetiva cotidiana de la red más amplia que la contiene.

La cultura como referencia holística en todo caso se reduce en la práctica a mensajes discretos, "prácticas de significado", o "discursos" los cuales son distribuidos por instituciones y medios de comunicación. ${ }^{23}$ La Universidad constituye una de esas instituciones y de allí su responsabilidad en la generación de los discursos que se convierten en producción cultural lista para ser convertida en práctica en cuanto es socializada. Aunque la producción cultural se concibe como un proceso de "hibridación" y "negociación"24, en espacios institucionales hegemónicos ésta puede imponerse sin mayores resistencias y resulta por lo tanto un mecanismo privilegiado para la reproducción del poder. La cultura que interesa a los estudios culturales es más que un objeto de estudio, pues es al mismo tiempo la localización de la crítica política y la acción. ${ }^{25}$

\section{La historia al debate}

Frente a las preocupaciones que hemos venido elaborando en este texto resulta apropiado preguntarse por su pertinencia frente al conocimiento histórico. Los historiadores, muy reacios a visualizar estas posibilidades de discusión, con una disciplina intelectual que según algunos tiene más de 2.400 años de edad, ${ }^{26}$ y con el cómodo tránsito entre el espacio reservado por la modernidad a las humanidades y

\footnotetext{
${ }^{22}$ Ibid., Xxiii.

${ }^{23}$ Simón During, ed., The Cultural Studies Reader (Londres: Routledge, 1993), 5.

${ }^{24}$ Ibid., 7.

${ }^{25}$ Ziauddin Sardar y Borin Van Loon, Cultural Studies for Beginners (Cambridge: Icón Books, 1997), 9.

${ }^{26}$ Keith Windschuttle, The Killing ofHistory: How Literary Critics and Social Theorists are Murdering our Past (New York: The Free, 1997), 1.
} 
el asignado a las ciencias sociales, se sienten muy seguros ante las crisis de fundamentación que hoy determina el desarrollo, o quizás el fin, de las ciencias.

En el ámbito colombiano el debate acerca de la crisis disciplinar y de los nuevos campos de conocimiento parece haberse atrasado un poco frente a la dinámica de otros países. ${ }^{27}$ Igualmente desafortunada es la adopción sin elaboración de alguna crítica a la posmodernidad, especialmente la de Fredric Jameson, ${ }^{28}$ que ha desvirtuado la discusión intelectual al crear simples focos de resistencia que aluden a la moda, la superficialidad, el carácter idealista o la ideología del capitalismo avanzado, todos epítetos que tomados en contextos simples, desvalorizan la riqueza de la crítica posmodernista, la cual merece ser tomada en cuenta aunque no se opte por ella. $\mathrm{Ni}$ hablar de las livianas reflexiones del físico Alan Sokal, que han tenido tanta divulgación en nuestro medio. ${ }^{29}$

No se trata aquí de repetir los debates acerca de la historia en la posmodernidad, limitados al contexto disciplinal. Los textos disponibles sobre el tema son ya innumerables y muchos de ellos poseen una consistencia a prueba de resistencias dogmáticas. Aunque el texto retoma la inspiración de los debates posmodernistas de los ochenta, su intencionalidad no es la de insistir en lo dicho. Se trata más bien de recordar algunas consecuencias posibles del debate para enfrentar las preguntas acerca de la fundamentación disciplinal que, en todo caso, no se limitan a una u otra expresión de la discusión entre modernistas y posmodernistas.

Se trata de explicitar y criticar el importante acento de nuestra historiografía en reproducir modelos demasiado tradicionales en la escritura de la historia, sin mencionar los efectos sociales que dicha escritura genera en el momento en que esta producción se convierte en insumo legitimador de la realidad.

\footnotetext{
${ }^{27}$ Es más, el tema parece haberse discutido, especialmente a escala latinoamericana, en la década del ochenta mientras que ahora la comunidad internacional se adentra en otros temas y corolarios de aquellos debates. Castro y Mendieta, Teorías sin disciplina.

${ }^{28}$ Fredric Jameson, El Posmodernismo o la Lógica Cultural del Capitalismo Avanzado (Barcelona: Paidós, 1991).

${ }^{29}$ Ver, por ejemplo, Alan Sokal y Jean Bricmont, Imposturas Intelectuales (Barcelona: Paidós, 1999).
} 


\section{FRONTERAS}

Como primer ejercicio me interesa sugerir unos pocos de muchos elementos posibles para desmitificar la solidez del ejercicio histórico. También me interesa sugerir aquí que, en lugar de ampliar la discusión disciplinal como lo hacen por ejemplo las subdisciplinas, podría pensarse en la fusión de la historia en los nuevos campos, redefiniéndola, verbigracia, como una versión de estudios culturales.

Así se negaría el estatuto exclusivo de la historia, como se ha entendido hasta ahora. La propuesta no es necesariamente nueva o alocada y de hecho, ya algunas universidades en el mundo han cambiado la adscripción institucional de sus departamentos de historia en otros de estudios históricos, dando un giro institucional importante frente a las tradiciones de la disciplinariedad, y varios académicos prominentes consideran que el estudio del pasado se hace mejor al aproximarse a las relaciones y prácticas sociales a través del análisis textual, más allá y de manera aún más radical que en las primeras propuestas del famoso "giro lingüístico" ${ }^{30}$.

La punta de lanza del cambio, en el caso de la historia, ha sido sobre todo el tema de lo cultural. Así, el primer campo de combate se ha dado alrededor de la historia cultural, pero aún allí me parece que la propuesta es tímida y podría proponerse un debate acerca de la cercanía de lo histórico con los estudios culturales, sacando la discusión del ámbito disciplinario. Claro, si la idea de que la historia cultural venía ejerciendo un imperialismo al considerar que toda la historia es historia cultural ${ }^{31}$, la resistencia que ofrecerá una referencia todavía más amplia a los estudios culturales, será sin duda enorme.

La solución, en todo caso, no sería una nueva historia, o una nueva interdisciplinariedad (caracterizada, como ya mencionamos, por fragmentos disciplínales que se reúnen), sino más bien el desarrollo de prácticas transdisciplinarias que superen realmente la fragmentación disciplinal hasta ahora vigente, para superar las limitaciones que impone la fragmentación del conocimiento.

La historia puede ser tratada con el mismo lenguaje crítico empleado en las otras disciplinas. Para ello, basta constatar su injustificada evasión de la discusión

\footnotetext{
${ }^{30}$ Windschuttle, The Killing of History, 15.

${ }^{31}$ Híspanc American Historical Review 79, no. 2 (mayo 1999): 204.
} 
epistemológica y una vez expuesta esta característica, ver cómo la crítica a la modernidad afecta a la historia tanto como a las ciencias sociales y por lo tanto su futuro se definirá en el debate común ante la aparición de los nuevos campos de estudio.

\section{A. El baluarte infranqueable}

Cuando se habla de crisis de la historia, ya sabemos que no hay unanimidad acerca de su carácter, que es el mismo de la crisis de las disciplinas, que es aquel de la crisis del pensamiento moderno ${ }^{32}$. Pero aquí no hablaremos de crisis en el sentido restringido que mira a situaciones problemáticas que al final generan avances en los paradigmas. Hablamos de crisis en el sentido de la credibilidad del canon y en la posibilidad de una transformación radical del conocimiento disciplinal.

La queja de Peter Novick de que en la historia "hay un sentimiento de desarraigo tal como no existe en ninguna otra especialidad" es común en todas las disciplinas. En eso la historia no tiene nada de especial. Es parte de la misma propuesta que se somete a crítica, esta es la de la disciplinariedad, la muralla que quiere preservar la objetividad y la autonomía de la disciplina.

Es cierto que la historia ha sido exitosa en su actividad empírica y en su desarrollo institucional en casi todos los países, y eso ha hecho pensar a algunos que los problemas actuales de la historia son solo una etapa más de su crecimiento lineal. El historiador francés Gérard Noiriel, por ejemplo, considera que transformaciones como la actual se vienen dando desde hace dos siglos; pero si uno recuerda a Thomas Khun y su influyente análisis acerca del desarrollo de las ciencias, queda claro que un problema que genera su solución dentro de un paradigma establecido no es lo mismo que las evidencias conducentes a una crisis pues esta última conlleva posiblemente a una ruptura y no a un simple avance en las diversas maneras de generar conocimiento.

Con estas actitudes, las revisiones historiográficas parecen quedarse en la descripción de las prácticas y en un análisis de las tendencias, pero sin llegar a confrontar

\footnotetext{
${ }^{32}$ En este tema de la crisis disciplinal véase una aproximación complementaria que discute el papel de la antropología y sus transformaciones más recientes en mi trabajo "¿Antropología Histórica... Antrohistoria...?". Memoria y Sociedad 3, no. 5 (agosto 1998): 147-60, del Departamento de Historia de la Universidad Javeriana.
} 
críticamente sus contenidos. A veces la crítica es simplemente una defensa de una manera de hacer historia, pero pocas veces se explicitan los contenidos de lo que Foucault llamaría el saber-poder en cada etapa de producción histórica.

Los historiadores siempre se han sentido en terreno firme en cuanto a su objeto, el pasado, pero basta recordar, por ejemplo, que cientos de libros recientes exploran los procesos históricos que hubieran podido ser si un evento hubiera sido diferente $^{33}$. Esta "historia alternativa" o "virtual" es un género establecido de literatura histórica que a veces se clasifica como ciencia ficción. A algunos esta producción les parecerá irrelevante; pero si uno mira con cuidado, su evaluación genera una pregunta central al quehacer tradicional de la historia: ¿cuáles son las causas necesarias de un evento? Además, de estos ejercicios se concluye que los hechos contradictorios son parte del ejercicio interpretativo. La publicación de las historias alternativas ciertamente ha retado las aproximaciones de la historia lineal y determinista y plantea el debate acerca de qué tan complejos y caóticos son los procesos históricos.

Por otra parte, aun sin el análisis de la alternatividad, en pasados que no sucedieron, el pasado que supuestamente sí existió puede ser igualmente problemático. Basta mirar la certeza de procesos de creación de falsas memorias en un evento que ya se conoce experimentalmente. En un reciente artículo de la revista Scientific American se da cuenta de diferentes casos en los cuales psiquiatras implantan falsa memorias en sus pacientes. En ocasiones estos casos han llegado a los tribunales indemnizando a los pacientes cuando éstos han actuado y afectado a otros en función de dichos recuerdos falsos ${ }^{34}$. Un número creciente de investigaciones demuestra que bajo las circunstancias adecuadas los recuerdos falsos pueden implantarse con mucha facilidad en las personas. De la misma manera, estos estudios demuestran cómo la información interesada puede cambiar el recuento que un individuo da de un hecho de manera predecible y a menudo muy poderosa. Cuando el tiempo pasa incluso los recuerdos originales se desvanecen y son suplantados por aquellos

\footnotetext{
${ }^{33}$ Ver Joñannes Bulhof, "What if? Modality and History", History and Theory 38, no. 2 (mayo 1999): 14568 y Aviezer Tucker, "Historiographical Counterfactuals and Historical Contingency," History and Theory 38, no. 2 (mayo 1999): 264-76.

${ }^{34}$ Elizabeth F. Loftus, "Creating False Memories", Scientific American (septiembre 1997): 71-75.
} 
recuerdos modificados a través de sugestión y otros mecanismos que incluyen la implantación de recuerdos sobre hechos que nunca sucedieron. El mecanismo más común es la sugestión inducida pero sobre todo cuando esta se convierte en un ejercicio de imaginación.

El acceso al pasado no es sólo difícil, es igualmente peligroso, cuando no pueden determinarse los hechos recordados y a qué ejercicios de sugestión o imaginación colectiva obedecen. Una primera aproximación a estos temas se encuentra en los trabajos de Hobsbawm y luego en los de Anderson, acerca de los mitos falsos ${ }^{35}$. Luego vino la rica producción acerca de "invenciones" que nos enseñaron de la construcción social del pasado. Los historiadores clásicos seguirán reivindicando la evidencia documental, pero después de los trabajos de Roger Chartier, ${ }^{36}$ por ejemplo, sabemos que el texto es construido de la misma manera que el testimonio oral y podemos añadir que está expuesto a las mismas inconsistencias, si se quiere, ontológicas, más allá de los procesos interpretativos, a pesar de todo el andamiaje metodológico tan preciado por los historiadores.

Así, el problema no sólo toca la dificultad del oficio de reconstruir discursivamente los hechos sino que pone en duda la tradicional aceptación ontológica de que el pasado existe independientemente de su apropiación por los historiadores.

Aunque se trate de las huellas del pasado que se recuperan, no todas ellas parecen relevantes. Se trata al tiempo de un problema ontológico y uno epistemológico. ¿El pasado es? ¿Qué importa al fin y al cabo? Lo que se decide está en la esfera del conocimiento, no en el de la existencia como tal; pero aun así esta seguridad ontológica sigue siendo preciada fuente de identidad para los historiadores.

En general, mi expectativa es que las preguntas sobre el quehacer histórico no pueden hacerse más desde el reducido espacio del oficio del historiador como se ha concebido tradicionalmente, y requieren otro aire, respirar más, buscar un espacio

\footnotetext{
${ }^{33}$ Ver, por ejemplo, Eric J. Hobsbawm, Sobre la Historia (Madrid: Crítica, 1999) y Benedict Anderson, Imagined Communities (Londres: Verso, 1983).

${ }^{36}$ Roger Chartier, El Mundo como Representación: Historia Cultural, entre Práctica y Representación (Barcelona: Gedisa, 1992).
} 
más integral que incluya aspectos esenciales de la filosofía, las humanidades y las ciencias sociales. No se trata de encontrar respuestas en la tradicional interdisciplinariedad que se presta los retazos, se trata de una preocupación epistemológica común a todas las ciencias, la cual sugiere sobrepasar las asfixiantes fronteras disciplínales.

\section{B. ¿Historia sin teoría?}

La atención a los temas de la profesionalización y de la institucionalización nos hacen olvidar a veces uno más esencial a la discusión global, éste es el de la fundamentación. Claro, ese tema no es muy atractivo para muchos historiadores engolosinados por el boom de la práctica histórica además porque sigue persistiendo un cierto escozor ante la teoría y no es raro ver que recurrentemente se encuentran prevenciones de historiadores por involucrar en sus escritos lo que despectivamente llaman la "aridez de la epistemología". ${ }^{37}$ Protegidos por una continuada adherencia al sentido común empírico y a las nociones realistas de representación y verdad, la mayoría de los historiadores han mantenido un desinterés y hasta una hostilidad hacia la filosofía que los ha llevado a aferrase a una ostensible posición ateórica o antiteórica ${ }^{38}$.

Como Nancy Partner, la prestigiosa medievalista, miembro del comité editorial de la revista History and Theory, nos recordaba el año pasado que "la historia nunca tuvo una teoría antes del posmodernismo aunque, por supuesto, sí tuvo aproximaciones a la filosofía". ${ }^{39}$ Igualmente, Gérard Noiriel, afirmaba que "la historia solo ha podido conquistar su autonomía en el ámbito intelectual situándose en el terreno de la investigación empírica y rechazando las generalidades sobre la historia que eran especialidad de los filósofos". ${ }^{40}$ En esta tradición lo que se establecía era una separación entre la investigación empírica y la especulación metafísica que planteaba la "filosofía de la historia". Pero esta última nada tiene que ver con la

\footnotetext{
${ }^{37}$ Gérard Noiriel, Sobre la Crisis de la Historia (Madrid: Cátedra, 1997), 11.

${ }^{38}$ Keith Jenkins, ed., The Postmodern History Reader (New York: Routledge, 1997), 1.

${ }^{39}$ Nancy Partner, "Truth and the Adequate Narrative: History after Postmodernism", en Social Sciences and Transdisciplinarity, 55-62.

${ }^{40}$ Noiriel, Crisis de la Historia, 51.
} 
reflexión teórica sobre el estatuto de la historia y su práctica, a lo cual corresponde la afirmación de una historia sin teoría.

Partner recuerda que "la filosofía de la historia ya fuera dialéctica, marxista o de cualquier otra ideología, característicamente ignoraba la escritura de la historia tomando por hecho en una aceptación total sin examinar la cosa precisa sobre la que tradicionalmente la filosofía trata-, la relación de las palabras con la realidad. Esto ya lo han repetido los posmodernistas hasta la saciedad, pero Partner agrega que es allí donde la "teoría" en el sentido ofrecido por el giro lingüístico se dirige a los propósitos para los cuales la filosofía fue inventada en primer lugar: examinar cómo el artefacto humano del lenguaje puede o no producir formas que cimentarán el conocimiento del mundo" . ${ }^{41}$ Así, el sentido de la teoría aquí no tiene que ver con un sistema ordenado de proposiciones probadas, como lo asumen muchos científicos sociales, ni tampoco es la versión del positivismo lógico como una explicación causal lógicamente integrada ${ }^{42}$.

Si se considera la historia desde el mundo griego y hasta los siglos posromanos de desarrollo europeo, la historia escasamente cambió. Siempre fue en prosa y fue una forma de producción literaria. No necesitó de una teoría ni atrajo mucho del examen filosófico o especulativo debido a que ella era solamente practicada, nunca enseñada. Por un breve momento cultural durante el final del siglo XIX y algunas décadas del XX, la historia se llamó a sí misma una ciencia y ganó reconocimiento como un tema universitario para la enseñanza y la investigación. Los historiadores practicaron y enseñaron su disciplina como una ocupación de tiempo completo, se profesionalizaron y crearon asociaciones, pero ninguna teoría científica propia acompañó este proceso de profesionalización. Algunos términos puntuales como factual, hipotético, evidencia, etc., fueron usados para describir la evaluación racional de las fuentes, pero eso fue lo más lejos que se llegó en términos de una autoconciencia científica. Una vez se evidenció que la historia no iba a dar lugar a las leyes y a los resultados replicables de los otros campos científicos, la historia

\footnotetext{
${ }^{41}$ Ibid.

${ }^{42}$ Craig Calhoun, Critical Social Theory: Culture, History and the Challenge of Difference (Cambridge: Blackwell, 1995), 5.
} 
abandonó esta pretensión, pero mantuvo su distancia de la literatura ignorando la actividad central de la escritura, tratándola como un accidente humano sin importancia necesario para la comunicación ${ }^{43}$.

El alejamiento de la filosofía, pero al tiempo la falta de éxito en la generación de leyes científicas, es lo que explica, según los miembros de la Comisión Gulbenkian, cómo en el trabajo de los historiadores se logró reflejar en el siglo XIX no sólo el dominio de la ciencia social nomotética en el pensamiento europeo, sino también las fuertes premisas de la actividad ideográfica y de actitudes antiteorizantes de una disciplina siempre ambigua entre las nuevas ciencias y las humanidades ${ }^{44}$.

La historia académica, insiste Partner, todavía tiende a identificarse con el lenguaje del taller, del trabajo de campo; todavía hablamos de "hacer historia", "llenar la brecha", "moldear" la historia, como si nuestros medios fueran cemento o metal, y no palabras. Los estudiantes de historia no necesariamente aprenden que son formados como escritores y que están trabajando en una disciplina literaria.

Aunque la aceptación del giro lingüístico, las prácticas del nuevo historicismo, y el debate acerca de la falta de teoría en el quehacer disciplinal son ya comunes, la resistencia es cada vez más notoria; pero también, y habría que ver por qué, apareció de manera un poco tardía. Quizá la evidencia de la transformación institucional fue percibida mucho después de que la crítica se clarificó como una tendencia hegemónica. La certeza acerca de los buenos fundamentos de la modernidad parecía ser un bastión irrebatible hasta que efectivamente se empezó a desmoronar en muchas Universidades.

Cómo síntesis de las agrias respuestas al cambio, recordemos uno de los libros más conocidos, The Killing of History, escrito por el historiador australiano Keith Windschuttle ${ }^{45}$. Este es un "texto belicoso en el cual el autor no toma prisioneros

\footnotetext{
${ }^{43}$ Ibid

${ }^{44}$ Wallerstein et al., Abrir las Ciencias Sociales, 16.

${ }^{45}$ Esta respuesta tiene su parangón en el lado de los dentistas naturales en un divulgado artículo de Alan Sokal quien después de su pilatuna de físico tuvo que convertirla en libro (citado más arriba) dada la difusión de su crítica que resultó más seria de lo que él mismo esperaba.
} 
cuando lanza su grito de guerra contra todos aquellos que han minado el prestigio de la ciencia histórica empírica." ${ }^{46} \mathrm{El}$ efecto de este tipo de libros es la polarización que hace desaparecer los espacios intermedios, incluso la posible continuidad que se pueda establecer en la arena del debate académico. El ataque a los sospechosos de posmodernismo, pero también a los tradicionalistas críticos, es una expresión clara de lo que podríamos llamar el pequeño escenario social dentro de la gran guerra de las ciencias.

Curiosamente las críticas de éste y otros libros parecidos hablan desde afuera de las coherencias que atacan, atribuidas a las "nuevas hordas" (no puedo resistir pensar en la analogía de lo bárbaro versus lo civilizado) ya sea por su irreverencia súbitamente descubierta, por su carácter de moda o aun por su lenguaje oscuro ${ }^{47}$.

Tristemente las discusiones epistemológicas de los historiadores rara vez alcanzan un nivel alto, y las posiciones filosóficas rápidamente se reducen a posiciones políticas simples. Y sólo de vez en cuando la resistencia de este tipo de autores adquiere visos de debate académico, como en la interesante crítica de Luc Ferry y Alain Renault en donde se ataca la "crítica total al mundo moderno" que desarrollan las "nuevas humanidades". Sin embargo, ésta, como la crítica de Víctor Farias, se concentra demasiado en la idea de que autores como Foucault, Derrida, Lacan e incluso Bourdieu, generan opciones "políticamente incorrectas." Esta posición explota incluso la conexión de Heiddeger, mentor intelectual de algunos de estos pensadores, con el nazismo, para demeritar enseguida su aporte intelectual. En realidad esta simpleza en la argumentación no resulta muy convincente y más bien parece indicar que la defensa proviene de todas partes menos desde la presunta solidez de los viejos estatutos. Más se parece al gran susto que genera constatar "un golpe dado por cortesanos disidentes dentro del mismo palacio". ${ }^{48}$

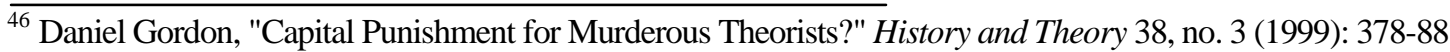

${ }^{47}$ Resulta interesante que el libro de Sokal antes referido reproduce el mismo esquema de ataque, desde una supuesta sobriedad del lenguaje y en contra de la parafernalia de las "nuevas humanidades".

${ }^{48}$ Windschuttle, The Killing of History, 9.
} 
FRONTERAS

delabistoria

Vol 5/2000

\section{Oportunidades para el cambio}

Una opción que me interesa a partir de la alternativa transdisciplinaria es aquella en la cual la historia se redefine como una versión de los estudios culturales más arriba discutidos. Se trataría de una nueva práctica en la que el eje interpretativo esencial es el análisis textual, pero en donde además su conexión con el presente asume una actitud altamente politizada.

La referencia, repito, es a los estudios culturales y no a una historia de la cultura. Esta distinción es importante y no siempre se resuelve con claridad. El peso de la formación pragmática de muchos colegas que hacen historia cultural muchas veces los repliega sobre la tradición del "oficio". Un caso ilustrativo aparece en uno de los textos más recientes acerca de la historia cultural en América Latina ${ }^{49}$. Allí se encuentran versiones cercanas a los estudios culturales, pero también aparecen los textos de otros historiadores que se ven casi arrastrados hacia diferentes campos, pero sin desordenarse y manteniendo férrea su ancla en "la evidencia que habla por sí sola". El espectro de la semiótica los ronda, pero, como Ulises parecen tapar sus oídos mientras cruzan por el texto como huyéndole al sonido encantador de las sirenas de la cultura. Más a diferencia de lo que hacen estos últimos autores, creemos aquí que no se trata de tomar argumentos de disciplinas vecinas para deformarlos y adaptarlos a necesidades e intereses de la disciplina propia. La vía propuesta es la contraria.

La entrada de las nuevas discusiones en América Latina a través de los historiadores ha sido más bien débil. Sólo en la década de los 90, un grupo de latinoamericanistas, cercanos a la academia de los Estados Unidos, empezó a descubrir las importantes similitudes de la Nueva Historia Cultural y las propuestas de los Grupos de Estudios Subalternos Asiáticos. El artículo seminal de Florencia Mallon que retomó la discusión promovida por Gilbert Joseph y complementada

\footnotetext{
${ }^{49}$ Entre los trabajos más relevantes mencionaré el número especial dedicado a la Nueva Historia Cultural en Méjico de la Hispanic American Historical Review 79, no. 2 (mayo 1999) y la excelente síntesis de trabajos editados por Gilbert M. Joseph, Catherine C. LeGrand y Ricardo Salvatore, Close Encounters of Empire: Writing the Cultural History of U.S.-Latin American Relations (Durham: Duke University Press, 1998).
} 
por Patricia Seed en torno a estos temas ${ }^{50}$ desarrolló un debate de gran importancia cuyos puntos conclusivos, por lo menos de parte del grupo rebelde, llevaron a publicar un manifiesto y a dedicar el número 2 de la revista Boundery al tema del posmodernismo en la historia de Latinoamérica.

Sin entrar en los detalles del debate a lo largo de los noventa, lo que me interesa aquí es recuperar las coincidencias de estos textos con el lenguaje expresado en la discusión alrededor del fin de las disciplinas, en sentido heideggeriano, en el cual la recuperación de una unidad del conocimiento dirigida a una diversidad factual enriquece la aproximación holística, al tiempo que se aparta de la pretensión universalista que tanto defienden los críticos de las nuevas tendencias.

A pesar de la constatación de esta revolución científica (o no tan científica a veces) el debate no va a resolverse en mucho tiempo. En otras palabras, mientras persistan las anteriores propuestas disciplinarias, las opciones transdisciplinarias solo pueden plantearse creando un "rancho aparte" que por ahora aparece como convivencia incómoda pero que inevitablemente se convertirá en combate por el control político de las universidades y en general de los espacios productores de conocimiento.

A los más ortodoxos, las propuestas holísticas que acompañan a las nuevas tendencias les seguirán pareciendo utópicas, producto de "modas intelectuales", y aún contraproducentes e irreales ante las crisis que vive el mundo. Pero, ciertamente los ejercicios del nuevo pensamiento son más que ideas. Son actividades intelectuales generadoras de nuevo sentido.

La falta de claridad en la lucha por los nuevos espacios ha creado interesantes cruces cuando aparecen, por ejemplo, instituciones defensoras de la tradición del

\footnotetext{
${ }^{50}$ Florencia Mallon, "The Promise and Dilemma of Subaltern Studies: Perspectives from Latin American History," American Historical Review 99, no. 5 (diciembre 1994): 1491-515; Gilbert Joseph, "On the Trail of Latin American Bandits: A Reexamination of Peasant Resistance", Latin American Research Review (en adelante citado como LARR) 25, no. 3 (1990): 7-53; Richard Slatta, "Bandits and Social Rural History: A Comment on Joseph," LARR 26, no. 1 (1991): 45-51; Gilbert Joseph, "'Resocializing' Latin American Banditry: A Reply," LARR 26, no. 1 (1991): 161-74; Patricia Seed, "Colonial and Postcolonial Discourse", LARR 26, no. 3 (1991): 181-200.
} 
conocimiento utilizando desprevenidamente a autores antidisciplinales que se infiltran sin resistencias. Recuerdo el muy reciente caso del Ministerio de Educación colombiano que, asociado con la UNESCO, publicó un texto del transdisciplinarista Edgar Morin, con pretensiones de apropiación institucional ${ }^{51}$. O se ve, la lenta pero fuerte presencia de nuevos institutos, encuentros y programas de estudios culturales, que se han incrustado en el corazón del cuerpo que combaten sin tregua. No se debe olvidar que una de las características de los nuevos campos es su compromiso político, de política académica, que busca con más o menos radicalidad la transformación de las instituciones universitarias. Se observa también el inmenso interés y el boom editorial de las nuevas tendencias, y se divisa finalmente el inevitable acercamiento de las recientes propuestas de pensamiento con los grupos externos a las universidades, al tiempo que crece en éstos el descontento con la academia clásica.

La sugerencia de un marco de análisis para construir regionalmente los espacios por fuera de las disciplinas, que revise los supuestos del latinoamericanismo e incluso proponga un análisis poslatinoamericanista, como lo discuten Santiago Castro y Eduardo Mendieta en texto arriba citado, requiere un primer paso y es el de criticar y desconstruir los contenidos de la ciencia en Latinoamérica.

Ante esta tarea, aparece un problema adicional que podría referirse como una especie de pobreza de la teoría reflejada en discursos inteligentemente pragmáticos, que no necesariamente renuevan la manera de dar sentido a la realidad y más bien la replican o por lo menos la alimentan de manera poco edificante y tienden a persistir largo tiempo legitimados por los sacerdotes del realismo, quienes consideran antiético "alejarse" de la dolorosa realidad de estos países. Cuando lo que se propone, al contrario, es una nueva forma de pensar esa realidad, precisamente ante la incapacidad del pensamiento moderno para enfrentarla adecuadamente.

Si el análisis del saber-poder para otras regiones del mundo presenta una cierta claridad, a pesar de su complejidad, en Colombia el aferramiento a la pretendida "realidad" genera precisamente la ausencia de proyectos políticos coherentes y

${ }^{51}$ Edgar Morin, Los Siete Saberes Necesarios para la Educación del Futuro (Bogotá: Ministerio de Educación Nacional, UNESCO, 2000). 
lleva a que la Universidad se desarrolle en una inercia donde las luchas de poder se realizan simplemente por el posicionamiento, sin saberse cuál será la práctica social efectiva de dicha postura. Esto replica el contexto de la lucha política, en Colombia por ejemplo, en donde el nivel de descomposición ideológica de los actores es enorme, actuando sin necesidad de referencias al proyecto político y casi exclusivamente en referencia a la estrategia militar.

En otras palabras, una labor de reconstitución disciplinal es una tarea política cuyos parámetros de poder están interpretados a la luz de las condiciones del mundo glocal (global-local), lo cual exige a cada uno de sus supuestos ontológicos y epistemológicos una revisión precisa a la luz de sus intencionalidades. Claro, la resistencia va más allá de los académicos disciplinaristas y se reproduce en las formas de ser hegemónicas, en todo el sentido dominador que proponía Gramsci.

No se trata necesariamente de desechar la búsqueda de las regularidades, o los matices hermenéuticos al nivel de la individualidad. Podría tratarse incluso de encontrar un camino intermedio en el cual la continuidad del conocimiento científico incluya el tema de la diversidad, se desarrolle entre la mirada regional y lo pertinente en el universo de las regularidades, y en el complemento entre la actividad nomotética y la ideográfica seguramente a partir de espacios disciplinares más flexibles, más holísticos y por supuesto más politizados.

Ha llegado quizá el momento de volver a reconocer que la teoría no está separada de la realidad concreta, a reivindicar una vez más la praxis del conocimiento. No sobraría que la teoría de sistemas hable de los desplazados de la violencia, que la arqueología del saber anteceda al análisis de las políticas públicas, que la hermenéutica potencie el trabajo participativo, que la crítica literaria traduzca los discursos burocráticos y sobre todo que la filosofía informe de nuevo a la historia, para empezar a reconstruir no sólo a las ciencias sino la identidad regional misma. Para saltar por encima de la propia sombra, tal vez sea pertinente apagar la luz del viejo conocimiento por un rato. 